\title{
A Parallel, Multi-Resolution Sensing Technique for Multiple Antenna Cognitive Radios
}

\author{
Nathan M. Neihart, Sumit Roy, and David J. Allstot ${ }^{1}$ \\ Dept. of Electrical Engineering \\ University of Washington \\ Seattle, WA 98195
}

\begin{abstract}
A parallel, multi-resolution spectrum sensing technique that is amenable to multiple-antenna cognitive radios is introduced. We show that for energy-detector-type spectrum sensors, the total sensing time due to FFT latency is reduced by 100 times using the proposed method versus the fixed-resolution, serial detection method employed in singleantenna systems. System-level tradeoffs such as the number of antennas, sensing bandwidth, and FFT size are also explored.
\end{abstract}

\section{INTRODUCTION}

Traditionally, radio transmitters are constrained to operate within a band of frequencies that has been set aside for their sole use by regulatory bodies (so-called licensed bands). But with many legacy technologies present, and with many new wireless standards on the horizon, the $1-10 \mathrm{GHz}$ spectrum is quickly becoming saturated. Recent measurements, however, show that actual spectrum usage varies between $15 \%$ and $85 \%$ based on the location and time of day [1]. This has led to the notion of 'cognitive' radios that are capable of recognizing unused frequencies, or "white spaces," and opportunistically using them until a licensed primary user needs them. The cognitive radio has the ability to move then to another unused frequency, thus using the available spectrum more efficiently. In this paper, we introduce a new multi-resolution parallel sensing algorithm for use with multiple-antenna OFDM transceivers and discuss system-level tradeoffs relating to sensing time.

\section{SPECTRUM SENSING CONSIDERATIONS}

An unlicensed cognitive radio enabled user in a given frequency band must not cause harmful interference to primary users. This requires that the cognitive radio be able to sense the available frequency spectrum for primary users, in addition to other cognitive radios, and decide upon a suitable transmission frequency. Moreover, once a suitable transmission frequency is found the cognitive radio must be capable of vacating that frequency as soon as a primary user is detected.

Typically, the total system bandwidth, $B_{S Y S}$, is large enough that it cannot be digitized and processed all at once. Hence, the system bandwidth must be processed in smaller blocks, each having a smaller bandwidth $B_{B L K}$. In order to adequately detect a primary user, the cognitive radio must have a minimum sensing frequency resolution of $F_{R E S}$, which is equal to $1 / 2$ the minimum bandwidth that can be occupied by a primary user. It is assumed here that the primary user can have variable bandwidths.

There are two architectural options for implementation of the sensing function. The first is to have a completely separate receive chain for spectrum sensing. This allows the radio to perform continuous spectrum sensing that results in immediate detection of new primary users, but at an undesirable cost in chip area and power consumption. A second, more practical solution, is to integrate the spectrum sensing hardware into the transceiver [2], [3], which results in a relatively small increase in area and power consumption as most of the sensing hardware is also used for data reception. In addition, with the proper choice of sensing period, which needs to be set by a regulatory body, the cognitive radio still avoids interfering with the primary user.

Given that the sensing hardware should be shared with the receiver as much as possible, two main methods for spectrum sensing have been described in the literature: energy detection [2], [4] and feature detection [5]. Energy detection is implemented by averaging frequency bins of an FFT. Feature detection, on the other hand, exploits the periodicity present in modulated signals. Although these sensing techniques differ in implementation, they have two characteristics in common: they sense $B_{S Y S} / B_{B L K}$ number of blocks in series and $B_{B L K}$ is the widest bandwidth that can be processed at a resolution of $F_{R E S}$ Furthermore, $B_{B L K}$ typically remains constant over the duration of the spectrum sensing. This leads to long sensing times and, consequently, a lower average data throughput. The average throughput is further degraded if the system bandwidth is large (e.g., 3-10GHz) or if the necessary sensing resolution must be very fine.

The total sensing time is reduced using a multi-resolution sensing technique wherein the total system bandwidth is first sensed using a coarse resolution. A fine resolution sensing is then performed over a small range of frequencies. This technique not only reduces the total number of blocks that must be sensed, it also allows the cognitive radio to avoid sensing the entire system bandwidth at the maximum resolution. Hur, et al. [6] propose a multi-resolution sensing technique in the analog domain using wavelets. In our

${ }^{1}$ Research supported by National Science Foundation contract CCR0086032, Semiconductor Research Corporation Contract 2006-HJ-1427, and a grand from the National Science Foundation Center for the Design of Analog/Digital Integrated Circuits (CDADIC). 
approach, an FFT-based energy detector is used for the reasons explained below.

In addition to multi-resolution sensing, parallel sensing can be employed to further reduce the total sensing time. It requires multiple data-chains at the receiver and, hence, is amenable to multiple-antenna receivers. In the case of an $M$ antenna receiver, the total sensing time is reduced by an approximate factor of $M$. Fig. 1 shows a block diagram of a multiple antenna receiver configured for both coarse ( Fig. 1(a)) and fine resolution sensing (Fig. 1(b)). In Fig. 1(a), a multi-tone frequency generator outputs four separate center frequencies. Each of the four down-converted frequency bands is digitized and fed into an $N / M$-point FFT block. Because this is coarse sensing, the size of the FFT can be small (i.e., the resolution can be large). The outputs of the four FFT blocks are input to a sensing block that determines the energy content in each of the four bands. This process continues until the entire system bandwidth has been sensed. At that point, the cognitive radio has determined which coarse resolution block has the least energy. The total number of coarse blocks that must be sensed per antenna in this way is:

$$
N_{A N T}=\frac{B_{S Y S}}{M B_{C R S}}
$$

where $B_{C R S}$ is the coarse sensing bandwidth.

When the radio has finished coarse resolution sensing, the block with the least energy content is then sensed again but at a fine resolution $\left(F_{R E S}\right)$ in order to detect white spaces and primary users. During the fine resolution sensing, all of the M-antennas are used to down-convert the same frequencies; likewise, all of the FFT resources are used to process this single bandwidth. By using multiple antennas to sense the same frequency, the spatial diversity helps make it possible to detect a primary user suffering from severe multipath fading or one that is "shadowed."

In order to compare the sensing time for the new parallel, multi-resolution sensing approach to the serial, fixedresolution approach, we first define the bandwidths of the coarse and fine resolution sensing modes, $B_{C R S}$ and $B_{F I N}$, respectively. $\mathrm{B}_{\mathrm{FIN}}$ is set by the number of points in the FFT, $N$, as well as the minimum sensing frequency resolution, $F_{R E S}$, and is given by:

$$
B_{F I N}=N F_{R E S} \text {. }
$$

The coarse resolution sensing bandwidth should be an integer multiple of $B_{F I N}$ and is given by:

$$
B_{C R S}=\alpha B_{\text {FIN }} \quad \text { where } \alpha=1,2,3, \ldots
$$

Substituting (2) and (3) into (1), the number of coarse sensing blocks that must be sensed per antenna is:

$$
N_{A N T}=\frac{B_{S Y S}}{\alpha N M F_{R E S}} .
$$

It is assumed that the FFT is implemented on a DSP with a clock operating at $F_{D S P}$. Further, it is assumed that the total number of multiplications and additions necessary to

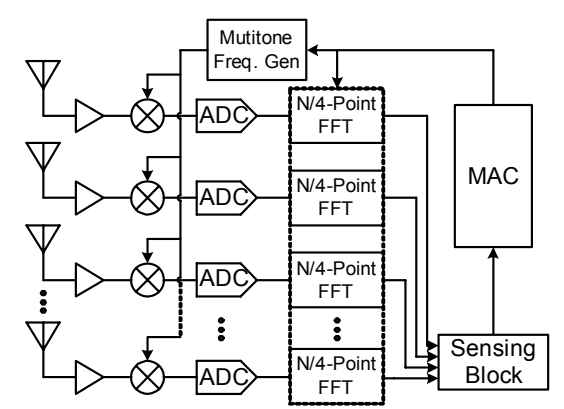

(a)

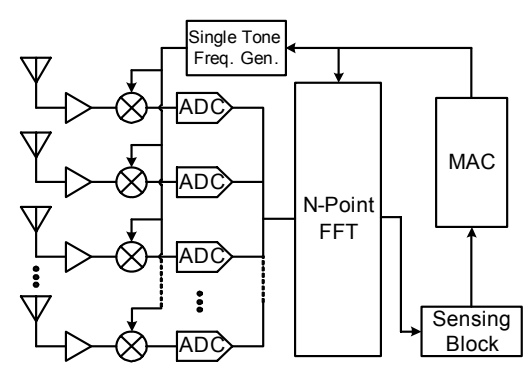

(b)

Figure 1. Block diagram showing a parallel, multi-resolution system configured for the (a) coarse resolution, and (b) fine resolution sensing modes.

perform a power-of-two N-point FFT is given by [7]:

$$
4 N \log _{2}(N)-6 N+8
$$

It is reasonable to expect that a given DSP can perform one multiplication and one addition per clock cycle. Hence, the approximate time spent coarsely sensing the total system bandwidth can now be written as:

$$
T_{C R S}=\frac{B_{S Y S}}{\alpha N M F_{R E S} F_{D S P}}\left[4 \frac{N}{M} \log _{2}\left(\frac{N}{M}\right)-6 \frac{N}{M}+8\right]
$$

Once a suitable bandwidth (of size $\alpha B_{F I N}$ ) has been found, the fine resolution sensing takes place in a serial fashion utilizing an N-point FFT as shown in Fig. 1(b). Again using (5) and realizing that the total number of blocks to be sensed at a fine resolution is $\alpha$, an equation can be written to approximate the time spent performing the fine resolution sensing:

$$
T_{F I N}=\frac{\alpha}{F_{D S P}}\left[4 N \log _{2}(N)-6 N+8\right] .
$$

The total time to perform the parallel, multi-resolution spectrum sensing is simply the sum of (6) and (7):

$$
\begin{aligned}
& T_{S}=\frac{B_{S Y S}}{\alpha N M F_{R E S} F_{D S P}}\left[4 \frac{N}{M} \log _{2}\left(\frac{N}{M}\right)-6 \frac{N}{M}+8\right] . \\
& +\frac{\alpha}{F_{D S P}}\left[4 N \log _{2}(N)-6 N+8\right]
\end{aligned}
$$


Note that (8) assumes that the switching time of the PLL is negligible when compared to the execution time of the Npoint FFT. Furthermore, this is in addition to the integration time required by the energy detector. In the next section, various system-level tradeoffs are considered and their effects upon $T_{S}$ are examined.

\section{SYSTEM-LEVEL CONSIDERATIONS}

Eqn. (8) is used to compare our new MIMO parallel, multi-resolution sensing technique to the single-antenna serial, fixed-resolution sensing method. First, however, various practical tradeoffs in the multiple antenna approach are explored such as the required number of antennas, the appropriate coarse resolution bandwidth, etc.

\section{A. Number of Antennas}

We first consider how the number of antennas impacts the sensing time. Examining (8), it is seen that $T_{S}$ is inversely proportional to $M$ and, hence, the system should have as many antennas as possible. If the transmitter is considered, more antennas imply higher data rates through spatial multiplexing. The disadvantage of increasing the number of antennas is a substantial penalty in chip area and power consumption due to the required replication of the transmit/receive chains. Moreover, the antennas must be spaced approximately $8-10$ wavelengths apart to ensure the received signals are uncorrelated, a requirement of both diversity and spatial multiplexing. This large spacing may be impractical for certain applications.

To maintain a balance between chip area, power consumption, data rate, and sensing performance, we constrain the maximum number of antennas to four. Fig. 2 shows a plot of $T_{S}$ versus number of antennas, $M$. The total system bandwidth is, $B_{S Y S}=1 \mathrm{GHz}$, the minimum resolution, $F_{R E S}=10 \mathrm{kHz}$, the FFT size, $N=64$, the coarse resolution bandwidth, $\alpha=10$, and $M$ ranges from 1 to 4 . The sensing time, $T_{S}$, decreases with increasing number of antennas. As the number of antennas becomes four, however, a point of diminishing returns is reached. At this point, the large increase in power consumption and chip area is not justified by a small decrease in sensing time. For this reason, four antennas are chosen for the system.

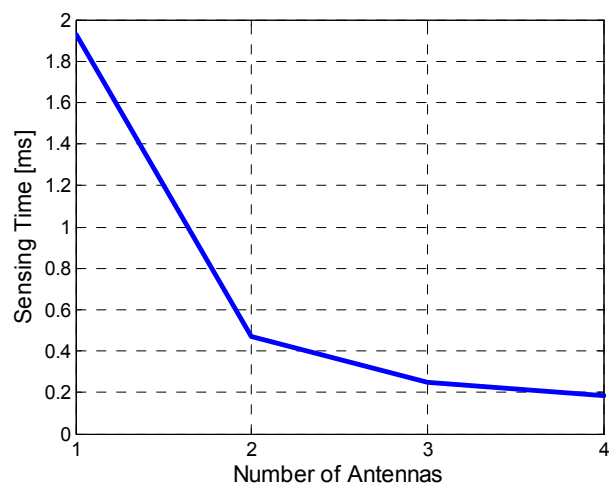

Figure 2. Sensing time, $T_{S}$, plotted versus the number of antennas, $M$. $B_{S Y S}=1 \mathrm{GHz}, F_{R E S}=10 \mathrm{kHz}, N=64, \alpha=10$.

\section{B. Coarse Resolution Sensing Bandwidth}

We next choose a proper bandwidth for the coarse resolution sensing mode as given by (3). Because the fine resolution sensing bandwidth is set in (2), the issue is merely a matter of choosing $\alpha$. Fig. 3 plots $T_{S}$ versus the number of antennas for different values of $\alpha$. Note that for a small number of antennas a large value of $\alpha$ is best and vice-versa. This is because for few antennas (e.g., $M=1$ ), a larger coarse resolution sensing bandwidth means that fewer overall blocks must be processed and, hence, fewer FFT results must be computed. For a larger number of antennas (e.g., $M=4$ ) parallelism reduces the number of serial frequency blocks that must be processed and, hence, as $\alpha$ increases beyond a point, it is merely adding more blocks that must be processed in the fine resolution sensing mode where the sensing is occurring at maximum resolution. For the final 4-antenna system, $\alpha$ is chosen to be 10 .

\section{Number of Points in FFT}

Because the final transceiver uses OFDM modulation, there are conflicting requirements for the FFT block: Transceiver flexibility on the one hand versus sensing time on the other. The FFT computation is one of the major contributors to sensing time which implies that the FFT should have as few points as possible (i.e., $N$ should be minimized in (8)). Fig. 4 shows a plot of sensing time versus the number of points in the FFT. The number of antennas is taken to be $4, \alpha=10$, $F_{R E S}=10 \mathrm{kHz}$, and $B_{S Y S}=1 \mathrm{GHz}$. Sensing time decreases almost linearly with $N$ until a point at which it begins to increase. For the case considered here, $N=4$ is the optimum number of points for the FFT.

One of the key attributes of a cognitive radio is flexibility; an OFDM transmitter is capable of transmission on two non-adjacent white spaces simply by turning off the sub-carriers that conflict with primary users. In order for this to work, the system needs as many sub-carriers as possible, which translates directly to maximizing $N$. Furthermore, more sub-carriers lead to higher data rates in an OFDM system.

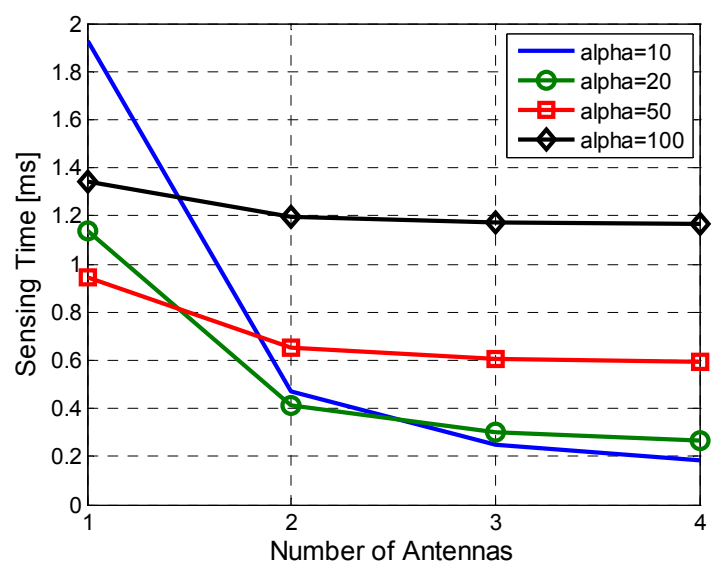

Figure 3. Sensing time, $T_{S}$, plotted versus the number of antennas for different values of $\alpha . B_{S Y S}=1 \mathrm{GHz}, F_{R E S}=10 \mathrm{kHz}, N=64$. 


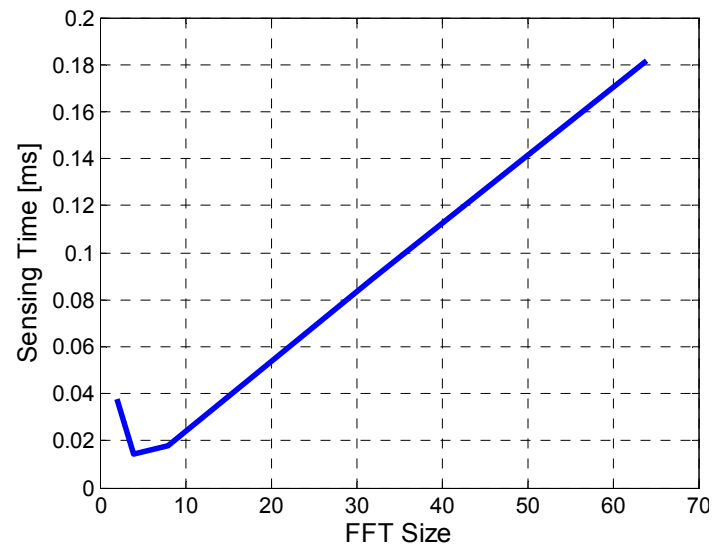

Figure 4. Sensing time, $T_{S}$, plotted versus the number of points in the FFT. $B_{S Y S}=1 \mathrm{GHz}, F_{R E S}=10 \mathrm{kHz}, M=4, \alpha=10$.

One possible solution to this is to use a variable $\mathrm{N}$-point FFT wherein a small number of points are used for sensing and a larger number of points are used for transmission and reception. The system described here assumes a fixed $\mathrm{N}$ point FFT with $N=64$.

\section{Comparison to a Single Serial Stream}

Fig. 5 shows (8) plotted versus the minimum resolution, $F_{R E S}$, for three different cases. Fig. 5(a) represents the parallel, multi-resolution sensing $(M=4)$ case; Fig. 5(b) shows the serial, multi-resolution $(M=1)$ case, and Fig. 5(c) plots the serial, fixed-resolution $(M=1)$ case. The parameters common to all three cases are: $B_{S Y S}=1 \mathrm{GHz}, N=64$, and $F_{R E S}$ varies between $10 \mathrm{kH}$ and $100 \mathrm{kHz}$. For the multi-resolution cases, $\alpha=10$.

Note that, as expected, the serial, multi-resolution case senses a $1 \mathrm{GHz}$ bandwidth ten times faster than the serial, fixed resolution case. Moreover, the parallel, multiresolution case senses the same $1 \mathrm{GHz}$ bandwidth 100 times faster than the serial, fixed resolution case, which clearly demonstrates the benefits of this approach.

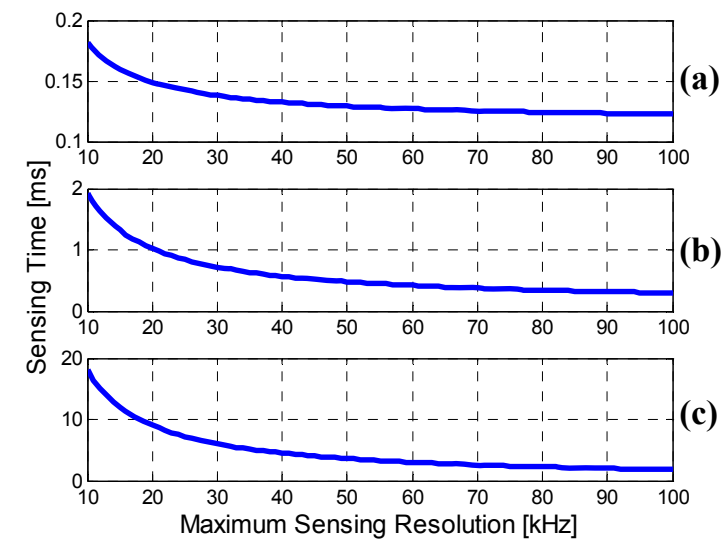

Figure 5. Sensing time, $T_{S}$, plotted versus minimum sensing resolution, $F_{R E S}$ for three cases. (a) parallel, multi-resolution case, $B_{S Y S}=1 \mathrm{GHz}, M=4, N=64, \alpha=10$; (b) serial, multi-resolution case, $B_{S Y S}=1 \mathrm{GHz}, M=1, N=64, \alpha=10$, and (c) serial, fixed-resolution case, $B_{S Y S}=1 \mathrm{GHz}, M=1, N=64$.

\section{CONCLUSIONS}

A new parallel, multi-resolution sensing technique has been shown in simulations to reduce the sensing time over the serial, fixed-resolution technique by approximately 100 times. Shortening the sensing time increases the average data throughput of the cognitive radio.

System-level tradeoffs are examined and their effects on sensing time. It is shown that for a large number of antennas (i.e., parallel paths), a smaller coarse resolution sensing bandwidth results in faster sensing times, whereas for a small number of antennas, a larger coarse resolution sensing bandwidth is preferred. Furthermore, while the number of points in the FFT gives more flexibility for an OFDM transceiver, it is better for sensing purposes to have fewer points in the FFT. It is also proposed to have a variable-point FFT with a low number of points during sensing and a high number of points during data transmission and reception.

\section{ACKNOWLEDGMENT}

The authors gratefully acknowledge the important contributions of Matt Miller and Pat Rakers of Freescale Semiconductor, Inc., and Prof. Jeyanandh Paramesh of Carnegie Mellon University.

\section{REFERENCES}

[1] FCC, Spectrum policy task force report, ET Docket No. 02-155, Nov. 2002.

[2] L. Jui-Ping, C. Po-An, and C. Tzi-Dar, "Design of a MIMO OFDM baseband transceiver for cognitive radio," ISCAS, pp. 4098-4101, May 2006.

[3] M. P. Wylei-Green, "Dynamic spectrum sensing by multiband OFDM radio for interference mitigation," DySPAN, pp. 619-625, Nov. 2005.

[4] H. Urkowitz, "Energy detection of unkown deterministic signals," Proc. of the IEEE, vol. 55, pp. 523-531, April 1967.

[5] D. Cabric, S. M. Mishra, R. W. Brodersen, "Implementation issues in spectrum sensing for cognitive radios," Conference on Signals, Systems and Computers, pp. 772-776, Nov. 2004.

[6] Y. Hur, J. Park, W. Woo, K. Lim, C.-H. Lee, H.S. Kim, and J. Laskar, "A wideband analog multi-resolution spectrum sensing (MRSS) technique for cognitive radio (CR) systems," ISCAS, pp. 4090-4093, May 2006.

[7] P. Duhamel, "Algorithms meeting the lower bounds on the multiplicative complexity of length- $2^{\mathrm{n}}$ DFTs and their connection with practical algorithms," IEEE Trans. Acoust. Speech. Sig. Proc., vol. 38, pp. 1504-1511, 1990. 